\title{
Relationship between Trunk Position Sense and Trunk Control in Children with Spastic Cerebral Palsy: A Cross-Sectional Study
}

\author{
Shilpa Monica $\mathbb{D}^{1},{ }^{1}$ Akshatha Nayak $\mathbb{D},{ }^{1}$ Abraham M. Joshua $\mathbb{D}^{1},{ }^{1}$ Prasanna Mithra, ${ }^{2}$ \\ Sampath Kumar Amaravadi $\mathbb{D}^{1,3}{ }^{1,3}$ Zulkifli Misri $\mathbb{D}^{4},{ }^{4}$ and Bhaskaran Unnikrishnan $\mathbb{D}^{2}$ \\ ${ }^{1}$ Department of Physiotherapy, Kasturba Medical College, Mangalore, Manipal Academy of Higher Education, Manipal, \\ Karnataka, India \\ ${ }^{2}$ Department of Community Medicine, Kasturba Medical College, Mangalore, Manipal Academy of Higher Education, Manipal, \\ Karnataka, India \\ ${ }^{3}$ Department of Physiotherapy, College of Health Sciences, Gulf Medical University, Ajman, UAE \\ ${ }^{4}$ Department of Neurology, Kasturba Medical College, Mangalore, Manipal Academy of Higher Education, Manipal, \\ Karnataka, India
}

Correspondence should be addressed to Akshatha Nayak; akshatha.nd@manipal.edu

Received 23 May 2020; Revised 25 June 2021; Accepted 5 August 2021; Published 21 August 2021

Academic Editor: Nicola Val

Copyright (C) 2021 Shilpa Monica et al. This is an open access article distributed under the Creative Commons Attribution License, which permits unrestricted use, distribution, and reproduction in any medium, provided the original work is properly cited.

\begin{abstract}
Background and Aim. Children with spastic cerebral palsy (CP) have impaired trunk control. Joint position sense, a component of proprioception, plays a crucial role in maintaining spinal mobility and stability. The current study is aimed at analysing the relationship between trunk control and trunk position sense in children with spastic CP. Methods. In this study, 24 children with spastic CP aged between 8 and 15 years were recruited. They were classified based on their functional performance using Gross Motor Function Classification System (GMFCS). Trunk control and trunk position sense were assessed using the trunk control measurement scale (TCMS) and digital goniometer, respectively. The correlation between these variables was tested using Spearman's correlation coefficient. Results. Significant negative correlation was found between trunk position sense and TCMS score. Similarly, a significant moderate correlation was found between trunk position sense and GMFCS. A strong negative correlation was also found between GMFCS and TCMS. Conclusion. Children with spastic CP with better trunk position sense had better trunk control. Similarly, children with higher functional performance had better trunk control and lesser error in trunk position sense. The current findings imply the relevance of proprioceptive training of the trunk for enhancing trunk motor control in children with spastic CP.
\end{abstract}

\section{Introduction}

Cerebral palsy is a nonprogressive developmental disorder due to injury to the developing brain, characterized by abnormalities in muscle tone, movement, and motor skills [1]. Abnormalities in tone and movement are responsible for poor postural control influencing daily functional activities such as sitting, reaching out, and walking $[2,3]$.

The trunk forms an initial frame for postural control, during postural stabilization and orientation; hence, it plays a vital role in performing goal-directed activities [4-7]. Trunk control, an active component of postural control, is a prerequisite for adequate, free, and specific mobility of the head and extremities [8-10]. Children with spastic cerebral palsy have poor trunk control owing to the weak trunk muscle strength, altered neural control, and inadequate position sense $[11,12]$. Literature review suggests a relationship between trunk control and sitting postural control, wherein children with CP present with impaired trunk control and poor maintenance of upright posture owing to impairments in anticipatory [13] and reactive $[14,15]$ postural responses and changes in ground reaction force during postural adjustment [16].

Topographically, trunk control is impaired significantly in quadriplegics followed by diplegics and lastly hemiplegics $[2,17]$. Also, children with quadriplegia manifest impaired 
static as well as dynamic trunk control, whereas children with diplegia and hemiplegia usually present with only an impaired static trunk control [2]. Maintaining trunk control while performing trunk movements in the sagittal plane is relatively easier than the frontal plane movements $[2,18]$. Based on the motor involvement, children in the lower Gross Motor Function Classification System (GMFCS) level had profound impairment of trunk control suggesting that there is a relationship between functional abilities and trunk control $[2,12]$. In addition, impaired trunk control in children with spastic CP is associated with balance dysfunction $[2,19]$.

Sensory deficits in children with CP, including proprioceptive dysfunction, can influence postural control [20, 21]. The joint position sense, a component of proprioception, increases body awareness and contributes to motor control and motor planning. Trunk position sense plays a crucial role in the maintenance of normal spinal mobility and stability [22] and helps in the development of motor skills [2] and postural control $[13,23]$. Altered trunk position sense has been established in various populations, such as poststroke hemiparesis people [11], balance-impaired older adults [24], and people with orthopaedic spine disease [22, 25, 26], but no studies discuss the impaired trunk position sense in children with CP.

The stabilization of the trunk is a core element to improve head stability, visual field orientation, and hand manipulation across a wide range of children with CP [27]. It is known that the trunk remains an essential component for the independent functioning of a child with a neurological disorder and children with CP have impaired selective control of the trunk affecting their ambulation and functional activities [28]. Literature review suggests several studies discussing the relationship between trunk position sense, balance, and postural control in various neurological conditions [11, 29, 30]. However, in children with spastic $\mathrm{CP}$, this relationship remains mostly unexplored. Therefore, this study was carried out to determine the relationship between the trunk position sense and trunk control in children with spastic CP.

\section{Materials and Methods}

2.1. Participants. A total of 24 children with spastic CP were recruited from the neurosensory developmental rehabilitation unit in Mangalore city. The Institutional Ethics Committee of Kasturba Medical College (Manipal Academy of Higher Education), Mangalore (IEC KMC MLR 11-13/224), approved the study. Children with all the types of spastic $\mathrm{CP}$ aged between 8 and 15 years, who were diagnosed with CP by paediatricians through MRI in GMFCS levels I-III, who could sit independently for 30 minutes, without back support and feet placed on the ground, and with Modified Mini-Mental Scale Examination score not less than 24, were included in the study [31]. Children were excluded if they had hearing or visual disabilities; if they had a history of other neurological, musculoskeletal, or medical conditions; and if they have been administered neural blockers like botulinum toxin and phenol in the last six months, interfering with the performance. In addition to the above, if the children experi- enced any pain during trunk movements during the assessment procedure, they were excluded from the study. All the eligible participants were approached; the objectives of the study were explained to their parents/legal guardians in a language they understood. A written informed assent from the participants and consent from the parents/legal guardians were obtained.

2.2. Procedure. The trunk control measurement scale (TCMS) was used to measure the trunk control. The trunk position sense was measured using a digital goniometer (Human Performance Measurement, Inc. Advanced Performance Meter 1 Digital Goniometer) in a supported standing position. Both trunk control and trunk position sense were assessed in a single contact session by the same trained therapist in a random order by a coin toss method to prevent any carryover effect of one assessment over the other. In total, the assessment was completed in a duration of 35 minutes to complete the entire assessment of a child with 15 minutes for trunk control assessment and 10 minutes to assess the trunk position sense. An additional 10-minute break was given between the two assessment measures to avoid the effect of fatigue over the second assessment measure.

2.2.1. Evaluation of the Trunk Control. Out of several tools available to measure trunk control, TCMS is proved to be a clinically comprehensive scale developed to assess the static and dynamic components of trunk control among children with spastic CP [17]. The TCMS comprises 15 test items measuring both static and dynamic components of the trunk control in sitting. The maximum value for the total TCMS is 58 points (20 for the category "static sitting balance," 28 for "selective movement control," and 10 for "dynamic reaching"). A higher TCMS score indicates better trunk control. The scale has acceptable interrater and intrarater reliability (interclass correlation coefficients $=0.91-0.99$; kappa $=$ $0.45-1$; Cronbach's alpha coefficients $=0.82-0.94$ ) and good validity (Spearman rank correlation between GMFM dimension B to E and TCMS, $r=0.82-0.94$ ) for assessing trunk control in children with spastic CP from 8 years to 15 years $[12,17]$. The standard technique was used to measure trunk control using TCMS [32].

2.2.2. Evaluation of the Trunk Position Sense. Active position sense of the trunk was assessed using a digital goniometer. Each participant was made to stand on a custom-made wooden frame with feet in a neutral position, shoulderwidth apart, knees extended (fastened with straps), hips relatively in neutral position, and pelvis strapped below the anterior superior iliac spine to decrease any proprioceptive feedback from the lower extremity and pelvis (Figure 1). The testing procedure was explained and two practice trials were given, where each participant was passively moved to the target position of $30^{\circ}$ trunk flexion; they were instructed to hold and memorize this position for 10 seconds with eyes open and also to understand the test procedure. Once the practice trials were completed, the participant was brought to the neutral position and was instructed to replicate the target position as accurately as he or she could with closed eyes. 


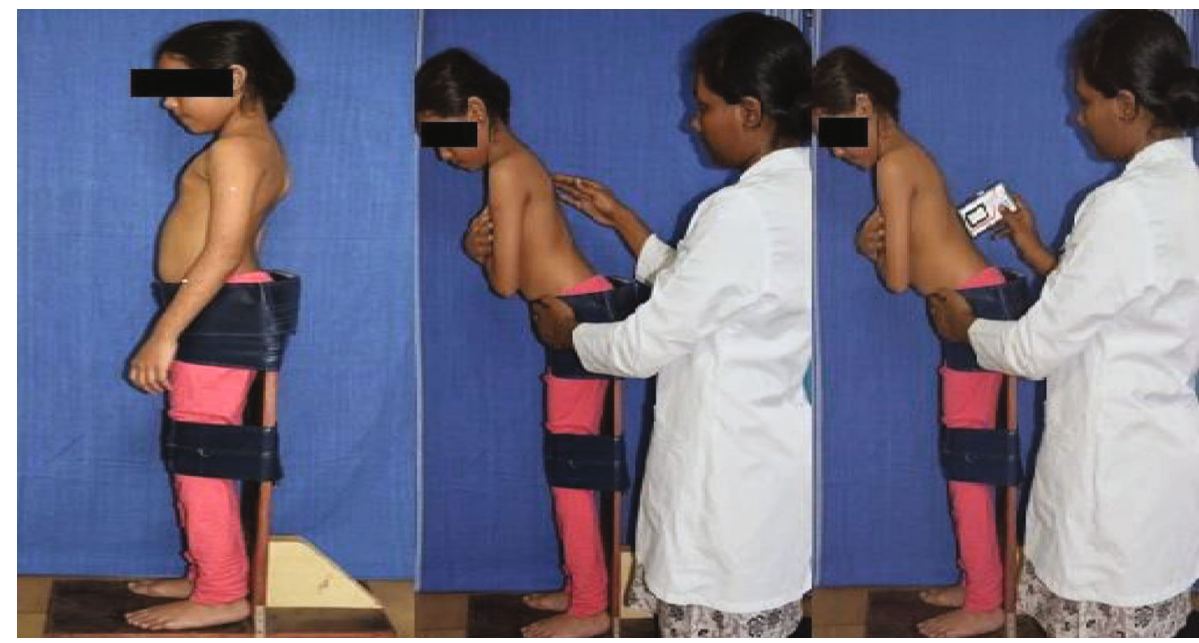

FIGURE 1: Measurement of trunk position sense.

Participant-perceived target position was measured using a digital goniometer with the reference point at the L1 lumbar spinous process.

No verbal or visual feedback or clues were given to the participants during the performance. All the participants could comply with the test without any adverse events. To add on further, considering the muscle strength impairments seen in CP, an angle of $30^{\circ}$ was chosen to test the trunk position sense; further increase in the range would have put additional load on the muscles. Inability to reposition the trunk to a greater flexion angle because of impaired muscle performance would adversely affect the test results.

2.3. Statistical Analysis. The collected data were coded and entered into Statistical Package for the Social Sciences (SPSS). The results were expressed as summary measures (median and interquartile range) and proportions using appropriate tables. The correlation of TCMS, trunk position sense, and GMFCS was done using Spearman's correlation coefficient as the data was not normally distributed, which was revealed on the Shapiro-Wilk test. A $p$ value of $<0.05$ was considered statistically significant. Correlation coefficient ( $r$ value) between 0.00 and 0.10 is considered as negligible correlation, 0.10 to 0.39 as weak correlation, 0.40 to 0.69 as moderate correlation, 0.70 to 0.89 as strong correlation, and 0.90 to 1.00 as very strong correlation [33].

\section{Results}

This study included 24 children with spastic CP, and their demographic characteristics are depicted in Table 1. The descriptive analysis of trunk position sense and TCMS scores, including median and interquartile range, is mentioned in Table 2 .

3.1. Relationship between the TCMS and Trunk Position Sense. The trunk position sense showed a moderate negative correlation with the total TCMS score $(r=-0.58, p=0.003)$ as well as static sitting balance and dynamic selective movement control subscale of TCMS, with a $p<0.05$ (Table 3 ).
TABle 1: Demographic data of the participants of the study $(n=24)$.

\begin{tabular}{lcc}
\hline & & $n(\%)$ \\
\hline \multirow{2}{*}{ Age group (8-15 yrs) } & 8 -10 yrs & $15(62.5)$ \\
Gender & $11-15$ yrs & $09(37.5)$ \\
& Male & $16(66.7)$ \\
Dominance & Female & $08(33.3)$ \\
& Right & $21(87.5)$ \\
Types of spastic cerebral palsy & Left & $03(12.5)$ \\
& Spastic hemiplegia-left & $02(8.3)$ \\
& Spastic triplegia & $01(4.1)$ \\
GMFCS levels & GMFCS I & $12(50)$ \\
& GMFCS II & $09(37.5)$ \\
& GMFCS III & $03(12.5)$ \\
\hline
\end{tabular}

n: number of subjects; GMFCS: Gross Motor Function Classification System.

However, dynamic reaching, another subscale of TCMS, showed a weak negative correlation with trunk position sense $(r=-0.35, p=0.098)$.

3.2. Relationship between GMFCS, Trunk Position Sense, and TCMS. When GMFCS levels were correlated with trunk position sense, a moderate significant positive correlation was found $(r=0.60, p=0.002)$ (Table 4$)$. A strong significant negative correlation was found (Table 4) when GMFCS levels were correlated with a total TCMS score $(r=-0.87, p<0.001)$.

\section{Discussion}

The present study is aimed at examining the relationship between trunk position sense and trunk control in children with spastic CP. Children with spastic hemiplegic and diplegic CP have previously exhibited good trunk control in static sitting conditions as this requires the trunk to be erect during quiet sitting and when the extremities are moved [18]. They also have fairly good control in forward-reaching tasks with challenges in lateral reaching and cross rotations [2]. The 
TABLE 2: Trunk position sense and TCMS mean scores of children with spastic CP.

\begin{tabular}{lccc}
\hline & Minimum & Maximum & Median [IQR] \\
\hline Trunk position sense (degree) & 1 & 8 & $4.5[2.75,6.00]$ \\
SSB $/ 20$ & 8 & 20 & $18[15.75,20.00]$ \\
D-SMC/28 & 9 & 25 & $22.00[12.75,25.00]$ \\
DR/10 & 5 & 10 & $10[8.75,10.00]$ \\
TCMS (total) $/ 58$ & 23 & 55 & $49[35.75,55.00]$ \\
\hline
\end{tabular}

IQR: interquartile range; SSB: static sitting balance; D-SMC: dynamic selective movement control; DR: dynamic reaching; TCMS: trunk control measurement scale.

TABLE 3: Correlation between trunk position sense and TCMS $(n=24)$.

\begin{tabular}{lcccc}
\hline & SSB & D-SMC & DR & Total score TCMS \\
$r(p$ value $)$ & $r(p$ value $)$ & $r(p$ value $)$ & $r(p$ value $)$ \\
\hline Trunk position sense & $-0.57\left(0.004^{*}\right)$ & $-0.62\left(0.001^{*}\right)$ & $-0.35(0.098)$ & $-0.58\left(0.003^{*}\right)$ \\
\hline${ }^{*}$ Significant. SSB: static sitting balance; D-SMC: dynamic selective movement control; DR: dynamic reaching; TCMS: trunk control measurement scale.
\end{tabular}

TABLE 4: Correlation between trunk position sense and TCMS with GMFCS levels $(n=24)$.

\begin{tabular}{lc}
\hline & GMFCS \\
& $r(p$ value $)$ \\
\hline Trunk position sense & $0.60\left(0.002^{*}\right)$ \\
TCMS & $-0.87\left(<0.001^{*}\right)$ \\
\hline
\end{tabular}

*Significant. GMFCS: Gross Motor Function Classification System; TCMS: trunk control measurement scale.

present study included primarily children with spastic diplegia, and a similar presentation was observed with respect to static sitting balance and dynamic reaching subscale. Abnormalities of selective movement control have been widely recognized as a primary manifestation of CP [34]. The movements of the trunk assessed in the dynamic selective movement control subscale are multiplanar, and the aforementioned loss of selective motor control could lead to poorer performance. The results of the present study revealed that children with spastic CP exhibited poor trunk control when evaluated using the TCMS, which is in agreement with the existing literature that investigated the trunk control in children with spastic CP [2]. This is the first study that evaluated trunk position sense in children with $\mathrm{CP}$ and revealed errors in repositioning the trunk which is higher than the normal individuals who are found to have an error of $2.6^{\circ}$ [35].

It was found that the individuals with impaired trunk position sense are able to perform motor tasks; however, the quality of the movement is compromised and the goaldirected actions execute lack of precision and postural responses, causing impaired balance and gait [36]. When trunk position sense was correlated with the subcomponents of trunk control, both static sitting balance subscale and dynamic selective movement control subscale showed a moderate significant negative correlation. In the static sitting balance subscale, the ability of the children to maintain stable sitting posture keeping the trunk erect when the limbs are being relatively moved is assessed, and during such a task where the body is in a stable position, the body primarily depends on the proprioceptive system for maintaining balance $[37,38]$. Hence, children with better trunk position sense perform better in the static sitting balance subscale. The test conditions in the dynamic selective movement control subscale consist of items where the ability of the children to move the trunk in different planes is assessed, which again primarily relies on the proprioceptive feedback from the body to execute the task [38].

Dynamic reaching subscale which tests the child's ability to reach both sideways and forwards beyond the limits of stability showed a weak negative correlation with trunk repositioning error. This could be because such conditions involve movements that require the use of feed-forward control where the anticipatory postural adjustments are made to maintain stability [36]. In the presence of inaccurate or reduced information from the somatosensory system, this feed-forward control relies more on the vestibular system [39].

In the current study, as the functional level increased on GMFCS, the error made with respect to trunk position sense decreased. The study findings are in line with the previous findings by Han et al. in which the children with lesser proprioceptive dysfunction had shown better motor performance, necessitating the role of proprioception for motor function [40]. Marked differences were found when GMFCS levels and TCMS in total and subscale scores were correlated. As the level of impairment increased on GMFCS, i.e., from level I to level III, the trunk control decreased, indicating a strong significant negative correlation of trunk control with GMFCS. The findings mentioned above suggest that trunk control is an essential component of functional abilities in CP children. This is in agreement with a study on impaired trunk control in spastic CP children, where trunk impairment was assessed based on the severity of motor involvement, which finally concluded that more significant deficits in trunk control were found in severely impaired children [2]. 
Though the study provides a unique understanding of trunk control impairment and trunk position sense, some critical reflections are warranted. The children included in the study were from GMFCS level I, level II, and level III. Hence, results cannot be generalized to all GMFCS levels. Children with spastic CP should be homogeneously grouped based on topography and GMFCS levels to find the appropriate correlation with clinical implications. Future studies encompassing trunk control, trunk position sense, and trunk muscle strength may contribute to a better understanding of the functional relationship in children with $\mathrm{CP}$.

\section{Conclusion}

In conclusion, the results of this study provide additional evidence of trunk control being impaired in spastic CP children. Children with spastic $\mathrm{CP}$ with better trunk position sense had better trunk control. Similarly, children with higher functional performance had enhanced trunk position sense and better trunk control. The present study findings also imply that therapeutic intervention focusing on proprioceptive training on an unstable surface and narrow base of support, which relay conflicting somatosensory feedback, may further improve trunk control in children with spastic CP.

\section{Data Availability}

The data used to support the findings of this study are available from the corresponding author upon request.

\section{Disclosure}

This research was a postgraduate dissertation in Kasturba Medical College, Mangalore, Manipal Academy of Higher Education, Manipal, India.

\section{Conflicts of Interest}

The authors declare no conflict of interest in this study.

\section{Acknowledgments}

We would like to thank the Manipal Academy of Higher Education for permitting us to do the research.

\section{References}

[1] S. Gulati and V. Sondhi, "Cerebral palsy: an overview," Indian Journal of Pediatrics, vol. 85, no. 11, pp. 1006-1016, 2018.

[2] L. Heyrman, K. Desloovere, G. Molenaers et al., "Clinical characteristics of impaired trunk control in children with spastic cerebral palsy," Research in Developmental Disabilities, vol. 34, no. 1, pp. 327-334, 2013.

[3] D.-H. Kim, D.-H. An, and W.-G. Yoo, "Changes in trunk sway and impairment during sitting and standing in children with cerebral palsy," Technology and Health Care, vol. 26, no. 5, pp. 761-768, 2018.

[4] F. Se, "Key factors in the development of lower limb co-ordination: implications for the acquisition of walking in children with cerebral palsy," Disability and Rehabilitation, vol. 25, no. 14 , pp. $807-816,2003$.
[5] G. Verheyden, A. Nieuwboer, L. De Wit et al., "Trunk performance after stroke: an eye catching predictor of functional outcome," Journal of Neurology, Neurosurgery \& Psychiatry, vol. 78, no. 7, pp. 694-698, 2007.

[6] G. Verheyden, L. Vereeck, S. Truijen et al., "Trunk performance after stroke and the relationship with balance, gait and functional ability," Clinical Rehabilitation, vol. 20, no. 5, pp. 451-458, 2006.

[7] C. Assaiante, S. Mallau, S. Viel, M. Jover, and C. Schmitz, "Development of postural control in healthy children: a functional approach," Neural Plasticity, vol. 12, no. 2-3, p. 118, 2005.

[8] S. Raine, L. Meadows, and M. Lynch-Ellerington, Bobath concepts: theory and clinical practive in neurological rehabilitation, John Wiley \& Sons, 2009.

[9] B. Bertenthal and C. von Hofsten, "Eye, Head and Trunk Control: The Foundation for Manual Development ${ }^{1}$," Neuroscience \& Biobehavioral Reviews, vol. 22, no. 4, pp. 515-520, 1998.

[10] S. Saavedra, M. Woollacott, and P. van Donkelaar, "Head stability during quiet sitting in children with cerebral palsy: effect of vision and trunk support," Experimental Brain Research, vol. 201, no. 1, pp. 13-23, 2010.

[11] S. Ryerson, N. N. Byl, D. A. Brown, R. A. Wong, and J. M. Hidler, "Altered trunk position sense and its relation to balance functions in people post-stroke," Journal of Neurologic Physical Therapy, vol. 32, no. 1, pp. 14-20, 2008.

[12] G. K. Seyyar, B. Aras, and O. Aras, "Trunk control and functionality in children with spastic cerebral palsy," Developmental Neurorehabilitation, vol. 22, no. 2, pp. 120-125, 2019.

[13] J. C. Van Der Heide, C. Begeer, J. M. Fock et al., "Postural control during reaching in preterm children with cerebral palsy," Developmental Medicine \& Child Neurology, vol. 46, no. 4, pp. 253-266, 2004.

[14] E. Brogren, H. Forssberg, and M. Hadders-Algra, "Influence of two different sitting positions on postural adjustments in children with spastic diplegia," Developmental Medicine \& Child Neurology, vol. 43, no. 8, pp. 534-546, 2001.

[15] P. Butler, S. Saavedra, M. Sofranac, S. Jarvis, and M. Woollacott, "Refinement, Reliability, and validity of the segmental assessment of trunk control," Pediatric Physical Therapy, vol. 22, no. 3, pp. 246-257, 2010.

[16] S.-F. Liao, T.-F. Yang, T.-C. Hsu, R.-C. Chan, and T.-S. Wei, "Differences in seated postural control in children with spastic cerebral palsy and children who are typically developing," American Journal of Physical Medicine \& Rehabilitation, vol. 82, no. 8, pp. 622-626, 2003.

[17] L. Heyrman, G. Molenaers, K. Desloovere et al., "A clinical tool to measure trunk control in children with cerebral palsy: the trunk control measurement scale," Research in Developmental Disabilities, vol. 32, no. 6, pp. 2624-2635, 2011.

[18] D. J. Curtis, P. Butler, S. Saavedra et al., "The central role of trunk control in the gross motor function of children with cerebral palsy: a retrospective cross-sectional study," Developmental Medicine \& Child Neurology, vol. 57, no. 4, pp. 351357, 2015.

[19] S. Panibatla, V. Kumar, and A. Narayan, "Relationship between trunk control and balance in children with spastic cerebral palsy: a cross-sectional study," Journal of Clinical and Diagnostic Research, vol. 11, no. 9, pp. YC05-YC08, 2017. 
[20] J. R. Wingert, H. Burton, R. J. Sinclair, J. E. Brunstrom, and D. L. Damiano, "Joint-position sense and kinesthesia in cerebral palsy," Archives of Physical Medicine and Rehabilitation, vol. 90, no. 3, pp. 447-453, 2009.

[21] M. J. Kurz, E. Heinrichs-Graham, D. J. Arpin, K. M. Becker, and T. W. Wilson, "Aberrant synchrony in the somatosensory cortices predicts motor performance errors in children with cerebral palsy," Journal of Neurophysiology, vol. 111, no. 3, pp. 573-579, 2014.

[22] E. E. Georgy, "Lumbar repositioning accuracy as a measure of proprioception in patients with back dysfunction and healthy controls," Asian Spine Journal, vol. 5, no. 4, pp. 201-207, 2011.

[23] R. Sæther and L. Jørgensen, "Intra- and inter-observer reliability of the trunk impairment scale for children with cerebral palsy," Research in Developmental Disabilities, vol. 32, no. 2, pp. 727-739, 2011.

[24] A. Goldberg, M. E. Hernandez, and N. B. Alexander, "Trunk repositioning errors are increased in balance-impaired older adults," The Journals of Gerontology. Series A, Biological Sciences and Medical Sciences, vol. 60, no. 10, pp. 1310-1314, 2005.

[25] M. Åsell, P. Sjölander, H. Kerschbaumer, and M. Djupsjöbacka, "Are lumbar repositioning errors larger among patients with chronic low back pain compared with asymptomatic subjects?," Archives of Physical Medicine and Rehabilitation, vol. 87, no. 9, pp. 1170-1176, 2006.

[26] A. Swinkels and P. Dolan, "Spinal position sense in ankylosing spondylitis," Spine (Phila Pa 1976), vol. 29, no. 4, pp. 413-420, 2004.

[27] V. Santamaria, J. Rachwani, S. L. Saavedra, and M. H. Woollacott, "Effect of segmental trunk support on posture and reaching in children with cerebral palsy," Pediatric Physical Therapy, vol. 28, no. 3, pp. 285-293, 2016.

[28] G. J. Barton, M. B. Hawken, R. J. Foster, G. Holmes, and P. B. Butler, "The effects of virtual reality game training on trunk to pelvis coupling in a child with cerebral palsy," Journal of Neuro Engineering and Rehabilitation, vol. 10, no. 1, p. 15, 2013.

[29] Ö. Onursal Kılınç, E. Ayvat, F. Ayvat et al., "The relationship between trunk position sense and postural control in ataxic individuals," Gait \& Posture, vol. 68, pp. 258-263, 2019.

[30] K. Jung, Y. Kim, Y. Chung, and S. Hwang, "Weight-shift training improves trunk control, proprioception, and balance in patients with chronic hemiparetic stroke," The Tohoku Journal of Experimental Medicine, vol. 232, no. 3, pp. 195-199, 2014.

[31] R. Moura, P. M. O. Andrade, P. L. B. Fontes et al., "Mini-mental state exam for children (MMC) in children with hemiplegic cerebral palsy," Dementia \& Neuropsychologia, vol. 11, no. 3, pp. 287-296, 2017.

[32] L. Heyrman, "Assessment of trunk control in sitting in children with cerebral palsy: the trunk control measurement scale," Developmental Medicine and Child Neurology, vol. 52, no. 4, p. 7, 2010.

[33] P. Schober, C. Boer, and L. A. Schwarte, "Correlation coefficients: appropriate use and interpretation," Anesthesia \& Analgesia, vol. 126, no. 5, pp. 1763-1768, 2018.

[34] R. Sæther, J. L. Helbostad, L. Adde, S. Brændvik, S. Lydersen, and T. Vik, "The relationship between trunk control in sitting and during gait in children and adolescents with cerebral palsy," Developmental Medicine \& Child Neurology, vol. 57, no. 4, pp. 344-350, 2015.
[35] L. Maffey-Ward, G. Jull, and L. Wellington, "Toward a clinical test of lumbar spine kinesthesia," The Journal of Orthopaedic and Sports Physical Therapy, vol. 24, no. 6, pp. 354-358, 1996.

[36] J. Konczak, D. M. Corcos, F. Horak et al., "Proprioception and motor control in Parkinson's disease," Journal of Motor Behavior, vol. 41, no. 6, pp. 543-552, 2009.

[37] P. T. Kyu-Bum Han, "Effects of trunk position sense through visual cue deprivation balance training in subacute stroke," Journal of the Korean Society of Physical Medicine, vol. 8, no. 3, pp. 327-335, 2013.

[38] S. H. Hosseinimehr and A. A. Norasteh, "The role of leg and trunk muscles proprioception on static and dynamic postural control," Citius Altius Fortius, vol. 26, no. 1, p. 5, 2010.

[39] E. B. Carlberg and M. Hadders-Algra, "Postural dysfunction in children with cerebral palsy: some implications for therapeutic guidance," Neural Plasticity, vol. 12, no. 2-3, p. 228, 2005.

[40] J. Han, G. Waddington, R. Adams, J. Anson, and Y. Liu, "Assessing proprioception: a critical review of methods," Journal of Sport and Health Science, vol. 5, no. 1, pp. 80-90, 2016. 